\title{
COMPARISON AND IDENTIFICATION OF FACTORS INFLUENCING THE FLAVORS OF ENTERAL NUTRITION AGENTS
}

\author{
NOBUYUKI WAKUI ${ }^{1 *}$, YURIKA ASHIZAWA ${ }^{1}$, NOBUTOMO IKARASHI ${ }^{2}$, YOSHIAKI MATIDA ${ }^{1}$, KAZUNORI WATANABE ${ }^{1}$
}

${ }^{1}$ Department of Faculty of Pharmaceutical Sciences, Division of Applied Pharmaceutical Education and Research, Hoshi University, Shinagawa, Japan. ${ }^{2}$ Department of Clinical Pharmacokinetics, Faculty of Pharmaceutical Sciences, Hoshi University, Shinagawa, Japan. Email: n-wakui@hoshi.ac.jp

Received: 16 January 2017, Revised and Accepted: 14 February 2017

ABSTRACT

Objective: It is important for medical professionals to understand the flavors of enteral nutrition formulas. For that, the flavors of enteral nutrition formulas were evaluated, and examined the factors influencing these flavors.

Methods: A total of 304 students in a pharmaceutical department were subjected to a semantic differential sensory evaluation in which they compared the flavors of digestion and semi-digestion enteral nutrition formulas using a five-point scale. In addition, factors related to good flavor were extracted through factor analyses, and subjected to covariance structure analysis (structural equation modeling).

Results: In the flavor comparison between digestion and semi-digestion nutrition formulas, semi-digestion nutrition agents scored significantly higher than digestion nutrition formulas did $(\mathrm{p}<0.001)$. The factor analyses extracted three subscales of factors related to good flavor: Impression of taking, feeling of the presence and sense of richness. In a path analysis model to determine the influence of these factors subscales on flavor, impression of taking and feeling of presence were found to have a significant influences $(\mathrm{p}<0.001)$.

Conclusion: It is important for medical professionals to understand the factors that influence the flavor, and thus provide patients with better nutrition formulas.

Keywords: Enteral nutrition, Medication adherence, Flavor, Nutritional status, Therapeutic efficiency.

(C) 2017 The Authors. Published by Innovare Academic Sciences Pvt Ltd. This is an open access article under the CC BY license (http://creativecommons. org/licenses/by/4. 0/) DOI: http://dx.doi.org/10.22159/ajpcr.2017.v10i5.17118

\section{INTRODUCTION}

In Japan, enteral nutrients, or artificially concentrated fluid nutritional supplements, are roughly classified by law as either food products or medical products, which are regulated by the Food Sanitation Act or Pharmaceutical Affairs Act, respectively [1]. Although the nutritional compositions of food products and medical products are very similar, differences in regulations have led to different manufacturing conditions for each. Specifically, the Food Sanitation Act does not allow the direct addition of microelements to enteral nutrients, and therefore, these supplements are manufactured from only natural or chemical compounds of the listed food additives. In addition, the Pharmaceutical Affairs Act requires the manufacturers of enteral nutrients to obtain approval for the production of medical products, and Japanese Pharmacopeia (JP) listed and JP unlisted drugs and the listed compounds of food additives can be added as ingredients [2]. Further differences between these products are related to clinical effects and research.

For example, pre-clinical data regarding medical properties and drug efficacies, as well as clinical data of healthy individuals and patients, are available for enteral nutrients classified as medical products, and thus the efficacies and safety of these products as medical drugs have been proven. In contrast, enteral nutrients classified as food products are considered under the same regulations as ordinary foods, and claims about their medical effects are forbidden according to the Pharmaceutical Affairs Act. Normally, these products are dispensed by nutritionists and are often used as a food material in the context of a hospital diet. In recent years, however, some of the enteral nutrients in the food category have been subjected to clinical testing in attempts to clarify their efficacies $[3,4]$.

Enteral nutrients in the food and medical categories also differ with respect to the financial burdens placed on patients. Although patients are required to cover all costs of enteral nutrients in the food category, medical categories enteral nutrients are covered by the national health insurance and can be available to patients for $<30 \%$ of the list price [2]. Accordingly, in Japan, where all citizens are covered by national health insurance, medical categories enteral nutrients, which place less of a financial burden on patients, are used more often.

These types of enteral nutrients also differ with respect to flavor. Specifically, those in the food category are said to taste better than those in the medical category. The reason for this difference is little restrictions on the manufacture of food categories enteral nutrition. Thus, allowing manufacturers to produce nutrient formulas with trendy or popular flavors. Importantly, the flavor of an enteral nutrient affects the patients' adherence to the take of these agents; in other words, a better flavor will thus improve patients' nutritional conditions and, ultimately, the effects of treatment on the primal pathologies. Enteral nutrients have been reported to significantly improve blood test value of patients [5]. In addition, rich diet induces an important gain in weight and leads to better recovery of gut mucosa in rats has been reported [6]. From this perspective, it is essential that medical professionals attempt to adequately understand the flavors of enteral nutrients to enhance patient adherence. However, flavors of enteral nutrients have not been compared for each type until now. Furthermore, factors that affect the taste of enteral nutrients have not been discussed yet. Accordingly, in this study, we conducted a sensory analysis using the semantic differential method [7] in which we compared the flavors of enteral nutrients and studied the factors that influenced these flavors.

\section{METHODS}

Types of enteral nutrition

Enteral nutrition formulas that are generally available in Japan were used for the sensory evaluation survey. The digestion nutrition agent Elental ${ }^{\circledR}$ 
(Ajinomoto Pharmaceuticals Co., Ltd, Tokyo, Japan), a compositional nutrition agent, was classified as a pharmaceutical preparation, whereas Pepuchino ${ }^{\circledR}$ (Terumo Corporation, Tokyo, Japan) was used as a food preparation. Regarding semi-digestion nutrition agents, Racol ${ }^{\circledR}$ NF combined enteral solution (Otsuka Pharmaceutical Factory, Inc., Tokushima, Japan) and Ensure ${ }^{\circledR} \mathrm{H}$ (Abbot Japan Co., Ltd, Tokyo, Japan) were used as pharmaceutical preparations, whereas Meibalance ${ }^{\circledR}$ (Meiji Co., Ltd., Tokyo, Japan) and Meibalance mini ${ }^{\circledR}$ (Meiji Co., Ltd., Tokyo, Japan) were used as food preparations. The evaluated flavors for each nutrition agent were as follows: Elental ${ }^{\circledR}$ : Coffee, pineapple, yogurt, tomato, grapefruit, mango, plum, bouillon, orange, and green apple; Pepuchino ${ }^{\circledR}$ : Lemon and apple; Ensure ${ }^{\circledR}$ H: Vanilla; Racol ${ }^{\circledR} \mathrm{NF}$, banana, vanilla, and coffee; Meibalance ${ }^{\circledR}$ : Vanilla; and Meibalance mini ${ }^{\circledR}$ : Coffee, caramel, yogurt, strawberry, banana, green tea, corn soup, and chocolate.

Regarding preparation, Elental ${ }^{\circledR}$, which is supplied in a powder form, was dissolved in water to a final concentration of $1 \mathrm{kcal} / \mathrm{ml}$. The attached special flavor was subsequently added at the recommended dose according to the Elental ${ }^{\circledast}$ product description. The original concentrations of liquid formulas were used (Pepuchino ${ }^{\circledR}$ : $1 \mathrm{kcal} / \mathrm{ml}$, Ensure ${ }^{\circledR} \mathrm{H}: 1.5 \mathrm{kcal} / \mathrm{ml}$, Racol ${ }^{\circledR} \mathrm{NF}: 1.0 \mathrm{kcal} / \mathrm{mL}$, Meibalance ${ }^{\circledR} 1.0: 1.0 \mathrm{kcal} / \mathrm{ml}$, and Meibalance $\mathrm{mini}^{\oplus}: 1.6 \mathrm{kcal} / \mathrm{ml}$ ).

\section{Design of a sensory evaluation based on the semantic differential method}

A total of 304 students in the pharmaceutical department were subject to a sensory evaluation based on the semantic differential method. The semantic differential method assesses which adjective of a bipolar pair more precisely describes the object of evaluation on a rating scale.

The following 20 evaluation pairs were selected to express the characteristics of the enteral nutrition formulas, according to a report by Mukai et al. [8,9]: (1) Sensation on the tongue; "gritty and unpleasant on the tongue/smooth and pleasant on the tongue," (2) taste; "strong flavor/weak flavor," (3) ease of consumption; "easy to drink/difficult to drink," (4) acridity; "weak acridity/strong acridity," (5) peculiar flavor; "no peculiar flavor/peculiar flavor," (6) freshness; "not consistently fresh flavor/always fresh flavor," (7) familiarity; "unfamiliar/familiar," (8) medicinal taste; "not medicinal/medicinal," (9) persistence of flavor; "no persistent flavor/persistent flavor," (10) meal similarity; "not meal-like/meal-like," (11) nutrition; "does not appear nutritious/appears nutritious," (12) consistency; "not fit for daily consumption/fit for daily consumption," (13) aftertaste; "weak aftertaste/strong aftertaste," (14) quality of flavor; "poor flavor quality/good flavor quality," (15) milky smell; "non-milky smell/milky smell," (16) greasiness; "not greasy/greasy," (17) positive mouthfeel; "dry and smooth/thick and mushy," (18) sensation when swallowed; "bad sensation when swallowed/good sensation when swallowed," (19) floury texture; "not floury/floury," and (20) flavor; "bad flavor/ good flavor." The test evaluation pairs were arranged such that a higher score indicated a better impression of the nutrition agent. The above pairs were assessed by semantic differential method with the following points: (1) Definitely, (2) probably, (3) neither, (4) probably, and (5) definitely (Fig. 1).

\section{Data analysis}

To evaluate flavor differences between nutrition agents, Welch's t-test was used for comparisons of mean differences between two groups, and a one-way analysis of variance (one-way ANOVA) was used for comparisons between three groups. If one-way ANOVA identified a significant difference, the Games-Howell multiple comparisons method was implemented as a post hoc test. Each score in each item is presented as a mean value \pm standard deviation (SD).

To identify factors that influenced flavor, factor analyses based on 19 evaluation pairs (excluding flavor) were implemented. A threefactor structure was adopted due to the attenuation condition of the eigenvalue and the possibility for factor interpretation. Factors were interpreted using Promax rotation, and those with eigenvalues $\geq 1$ were included as common factors in the maximum likelihood estimation. In addition, Spearman's correlation analysis was implemented to compare common factors identified in the factor analysis with good flavor to interpret how each common factor contributed to good flavor. Subsequently, covariance structure analysis (structural equation modeling [SEM]) was used for a confirmatory factor analysis based on the acquired results. Path analysis is the statistical technique used to examine causal relations between variables of the specialized model. We used four indices to evaluate the optimum model: The goodnessof-fit index (GFI), the adjusted GFI (AGFI), the root mean square error of approximation (RMSEA), and the Akaike information criterion (AIC).

The significance level for all tests was set at $\mathrm{p}=0.05$. For data analyses, one-way ANOVA and multiple comparisons were used. For factor analyses, IBM ${ }^{\circledR}$ SPSS statistics ${ }^{\circledR} 22$ software (IBM SPSS, Tokyo, Japan) was used. For the covariance structure analysis, IBM ${ }^{\circledR}$ SPSS Amos $^{\circledR}$ 22 software (IBM SPSS, Tokyo, Japan) was used.

\section{RESULTS}

Aggregate results for each evaluation pair

The mean values and SDs of the 20 survey pairs in the sensory evaluation were calculated, and the score distribution was verified

\begin{tabular}{|c|c|c|c|c|c|c|c|}
\hline \multicolumn{8}{|c|}{ Semantic differential method pairs, for expressing the characteristics of the enteral nutrition } \\
\hline & & Definitely & Probably & Neither & Probably & Definitely & \\
\hline (1) sensation on the tongue & gritty and unpleas ant on the tongue & 1 & 2 & 3 & 4 & 5 & smooth and pleas ant on the tongue \\
\hline (2) taste & strong flavor & 1 & 2 & 3 & 4 & 5 & weak flavor \\
\hline (3) ease of consumption & difficult to drink & 1 & 2 & 3 & 4 & 5 & easy to drink \\
\hline (4) acridity & strong acridity & 1 & 2 & 3 & 4 & 5 & weak acridity \\
\hline (5) peculiar flavor & peculiar flavor & 1 & 2 & 3 & 4 & 5 & no peculiar flavor \\
\hline (6) freshness & not consistently fresh flavor & 1 & 2 & 3 & 4 & 5 & always fresh flavor \\
\hline (7) familiarity & unfamiliar & 1 & 2 & 3 & 4 & 5 & familiar \\
\hline \begin{tabular}{|l|} 
(8) medicinal taste \\
\end{tabular} & not medicinal & 1 & 2 & 3 & 4 & 5 & medicinal \\
\hline (9) persistence of flavor & no persistent flavor & 1 & 2 & 3 & 4 & 5 & persistent flavor \\
\hline (10) meal similarity & not meal-like & 1 & 2 & 3 & 4 & 5 & meal-like \\
\hline (11) nutrition & does not appear nutritious & 1 & 2 & 3 & 4 & 5 & appears nutritious \\
\hline (12) consistency & not fit for daily consumption & 1 & 2 & 3 & 4 & 5 & fit for daily consumption \\
\hline \begin{tabular}{|l|} 
(13) aftertaste \\
\end{tabular} & strong aftertaste & 1 & 2 & 3 & 4 & 5 & weak aftertaste \\
\hline (14) quality of flavor & poor flavor quality & 1 & 2 & 3 & 4 & 5 & good flavor quality \\
\hline (15) milky smell & milky smell & 1 & 2 & 3 & 4 & 5 & non-milky smell \\
\hline (16) grea siness & greasy & 1 & 2 & 3 & 4 & 5 & not greasy \\
\hline (17) positive mouthfeel & thick and mushy & 1 & 2 & 3 & 4 & 5 & dry and smooth \\
\hline (18) sensation when swallowe & bad sensation when swallowed & 1 & 2 & 3 & 4 & 5 & good sensation when swallowed \\
\hline \begin{tabular}{|l|} 
(19) floury texture \\
\end{tabular} & floury & 1 & 2 & 3 & 4 & 5 & not floury \\
\hline (20) flavor & bad flavor & 1 & 2 & 3 & 4 & 5 & good flavor \\
\hline
\end{tabular}

Fig. 1: List of evaluation pairs used in the semantic differential method 
(Table 1). Although score deviations were observed for some items, all items were found to include important information needed to understand the flavor profiles of enteral nutrition agents and were thus subject to the analyses.

Flavor comparison between digestion nutrition agents and semidigestion nutrition agents

Mean scores of digestion nutrition and semi-digestion nutrition agents were compared. The mean value for Meibalance mini ${ }^{\circledR}, \mathrm{Racol}^{\circledR} \mathrm{NF}$, Ensure $^{\circledR} \mathrm{H}$, and Meibalance ${ }^{\circledR}$, which comprised the semi-digestion nutrition agent group $(n=533)$, was $3.15 \pm 1.38$. The mean value for Elenta ${ }^{\circledR}$ and Pepuchino ${ }^{\circledR}$, which comprised the digestion nutrition agent group $(\mathrm{n}=538)$, was $2.31 \pm 1.11$. A comparison of the mean values indicated that the semi-digestion nutrition group received a significantly higher mean score than the digestion nutrition agent group did (Welch's t-test, $\mathrm{p}<0.001$ ).

\section{Comparison of the flavor factor}

Nutrition agents were then compared with respect to good flavor. The following mean values were obtained (in decreasing order): Meibalance mini ${ }^{\circledR}$ - 3.69 \pm 1.21 ; Racol $^{\circledR} \mathrm{NF}$ - 3.08 \pm 1.40 ; Ensure ${ }^{\circledR}$ H - 2.87 \pm 1.33 ; Elental $^{\circledR}-2.57 \pm 1.15$; Meibalance ${ }^{\circledR}-2.20 \pm 1.33$; and Pepuchino $^{\circledR}$ - 2.06 \pm 1.00 . One-way ANOVA revealed significant differences among these agents $(\mathrm{p}<0.001)$. The Games-Howell multiple comparison post hoc analysis revealed that Meibalance mini ${ }^{\circledast}$, in particular, received a significantly higher value than all other nutrition agents (each $\mathrm{p}<0.001$ ).

\section{Extraction of factors affecting flavor via factor analysis}

Following the sensory evaluation, factor analyses were implemented using 19 evaluation pairs (excluding good taste), as described in the methods. Based on a factor load of 45, seven evaluation pairs (acridity, freshness, sensation when swallowed, floury texture, nutrition, meal similarity, and sensation on the tongue) with lower factor loads were eliminated from the analysis. The remaining 12 pairs were again subjected to factor analysis involving a maximum likelihood estimation and Promax rotation. Table 2 shows the final results of the factor analysis after Promax rotation. Three factors were extracted. The sum of three factors loading which was explained after the factor extraction against all distribution for 12 evaluation pairs was $52.16 \%$. In this study, Factor I comprised five adjective pairs to evaluate the quality of flavor, familiarity, ease of consumption, consistency, and medicinal taste. This factor was designated "impression of taking" because it was loaded with factors strongly related to the impression of taking. Factor II comprised three pairs to evaluate, greasiness, milky smell, and positive mouthfeel. This factor was designated "sense of richness." Factor III comprised four pairs to evaluate persistence of flavor, taste, aftertaste, and peculiar flavor. This factor was designated "feeling of presence."

\section{Correlation between the three identified factors and good flavor} The mean scores of the three factors extracted through factor analysis were calculated, and the correlations between each factor and good flavor were explored. Mean scores of $2.73 \pm 1.04,3.14 \pm 1.14$, and $2.41 \pm 0.90$ were obtained for the impression of taking, sense of richness, and feeling of presence, respectively. Cronbach's alpha was used to evaluate internal consistency and yielded adequate values of $\alpha=0.845$, 0.756 , and 0.722 for the impression of taking, sense of richness, and feeling of presence, respectively.

In the correlation analysis of the impression of taking, sense of richness, and the feeling of presence with flavor, a strong positive correlation $(r=0.792, p<0.001)$ was observed between flavor and impression of taking. Weak positive correlations were observed between flavor and the feeling of presence $(\mathrm{r}=0.251, \mathrm{p}<0.001)$, the impression of taking and feeling of presence $(r=0.363, p<0.001)$, and the sense of richness and the feeling of presence $(r=0.383, p<0.001)$. In contrast, a weak negative correlation was observed between flavor and the sense of richness ( $\mathrm{r}=0.89, \mathrm{p}=0.003$ )

\section{Identification of factors that influence flavor}

To assess the influences on flavor of the three-factor subscales in the sensory evaluation survey, a covariance structure path analysis was implemented using mean values acquired via the summation of all nutrition agent $(\mathrm{n}=1071)$ scores from each subject, without distinguishing the type of nutrition agent.

Using the results in Table 2, an analysis was implemented that assumed the influence of all three factors on flavor. As a result, the path coefficient from the sense of richness to flavor was not significant, and the index of the goodness of fit revealed the following: $\chi^{2}=474.558, \mathrm{df}=61$, $\mathrm{p}<0.001, \mathrm{GFI}=0.938, \mathrm{AGFI}=0.907$, RMSEA $=0.080$, and AIC $=534.558$. A subsequent analysis was implemented after deleting the insignificant path, after which AIC decreased to 532.883 to yield the final model (goodness of fit: $\chi^{2}=474.883, \mathrm{df}=62, \mathrm{p}<0.001, \mathrm{GFI}=0.938, \mathrm{AGFI}=0.909$, RMSEA=0.079; Fig. 2).

Table 1: Mean values and SDs for the 20-item sensory evaluation of six types of enteral nutrition

\begin{tabular}{|c|c|c|c|c|c|c|c|}
\hline \multirow[t]{2}{*}{ Sensory evaluation items } & \multicolumn{7}{|c|}{ Types of enteral nutrients } \\
\hline & Elental & Peptino & Ensure H & Maybalance mini & Racol-NF & Maybalance & Total \\
\hline Sensation on the tongue & $3.03 \pm 1.09$ & $3.25 \pm 1.19$ & $2.80 \pm 1.14$ & $3.32 \pm 1.09$ & $3.11 \pm 1.15$ & $2.58 \pm 1.00$ & $3.09 \pm 1.14$ \\
\hline Ease of consumption & $2.78 \pm 1.31$ & $2.33 \pm 1.21$ & $2.79 \pm 1.29$ & $3.49 \pm 1.24$ & $3.13 \pm 1.30$ & $2.17 \pm 1.26$ & $2.80 \pm 1.33$ \\
\hline Acridity & $3.18 \pm 1.31$ & $3.03 \pm 1.28$ & $3.26 \pm 1.27$ & $3.57 \pm 1.19$ & $3.33 \pm 1.15$ & $2.65 \pm 1.23$ & $3.21 \pm 1.27$ \\
\hline Peculiar favor & $2.51 \pm 1.33$ & $2.52 \pm 1.23$ & $2.30 \pm 1.17$ & $2.87 \pm 1.21$ & $2.39 \pm 1.20$ & $1.97 \pm 1.12$ & $2.50 \pm 1.25$ \\
\hline Freshness & $2.47 \pm 1.20$ & $2.50 \pm 1.23$ & $2.20 \pm 1.16$ & $2.75 \pm 1.20$ & $2.52 \pm 1.24$ & $2.23 \pm 1.18$ & $2.48 \pm 1.21$ \\
\hline Familiarity & $2.58 \pm 1.31$ & $2.27 \pm 1.20$ & $2.89 \pm 1.29$ & $3.42 \pm 1.25$ & $3.12 \pm 1.34$ & $2.17 \pm 1.26$ & $2.74 \pm 1.34$ \\
\hline Medicinal taste & $3.06 \pm 1.33$ & $2.70 \pm 1.35$ & $3.65 \pm 1.15$ & $3.67 \pm 1.20$ & $3.46 \pm 1.29$ & $2.48 \pm 1.28$ & $3.19 \pm 1.34$ \\
\hline Persistence of flavor & $2.52 \pm 1.28$ & $2.79 \pm 1.33$ & $1.94 \pm 1.07$ & $2.75 \pm 1.23$ & $2.43 \pm 1.24$ & $2.00 \pm 1.09$ & $2.50 \pm 1.28$ \\
\hline Meal similarity & $2.16 \pm 1.19$ & $1.96 \pm 1.02$ & $2.80 \pm 1.23$ & $2.84 \pm 1.35$ & $2.73 \pm 1.39$ & $2.58 \pm 1.24$ & $2.42 \pm 1.27$ \\
\hline Nutrition & $2.56 \pm 1.19$ & $2.94 \pm 1.23$ & $2.25 \pm 1.15$ & $2.56 \pm 1.14$ & $2.38 \pm 1.15$ & $2.27 \pm 1.12$ & $2.57 \pm 1.20$ \\
\hline Consistency & $2.24 \pm 1.34$ & $1.93 \pm 1.16$ & $2.12 \pm 1.23$ & $2.96 \pm 1.42$ & $2.43 \pm 1.37$ & $1.90 \pm 1.22$ & $2.28 \pm 1.34$ \\
\hline After taste & $2.24 \pm 1.23$ & $2.42 \pm 1.29$ & $1.98 \pm 1.09$ & $2.52 \pm 1.16$ & $2.27 \pm 1.11$ & $2.13 \pm 1.13$ & $2.30 \pm 1.20$ \\
\hline Quality of favor & $2.56 \pm 1.21$ & $2.12 \pm 1.14$ & $2.87 \pm 1.29$ & $3.33 \pm 1.27$ & $2.87 \pm 1.31$ & $2.43 \pm 1.37$ & $2.67 \pm 1.30$ \\
\hline Milky smell & $3.87 \pm 1.29$ & $4.03 \pm 1.15$ & $2.08 \pm 1.12$ & $2.59 \pm 1.21$ & $2.27 \pm 1.09$ & $1.92 \pm 0.91$ & $3.12 \pm 1.45$ \\
\hline Positive mouthfeel & $3.21 \pm 1.33$ & $3.67 \pm 1.30$ & $2.32 \pm 1.18$ & $2.75 \pm 1.17$ & $2.73 \pm 1.12$ & $2.52 \pm 1.10$ & $3.01 \pm 1.32$ \\
\hline Sensation when swallowed & $2.60 \pm 1.16$ & $2.74 \pm 1.24$ & $2.41 \pm 1.01$ & $2.83 \pm 1.10$ & $2.77 \pm 1.15$ & $2.17 \pm 0.91$ & $2.64 \pm 1.15$ \\
\hline Floury texture & $3.62 \pm 1.24$ & $4.17 \pm 1.04$ & $3.62 \pm 1.21$ & $3.63 \pm 1.14$ & $3.32 \pm 1.28$ & $3.30 \pm 1.32$ & $3.71 \pm 1.21$ \\
\hline Flavor & $2.57 \pm 1.15$ & $2.06 \pm 1.00$ & $2.87 \pm 1.33$ & $3.69 \pm 1.21$ & $3.08 \pm 1.40$ & $2.20 \pm 1.33$ & $2.73 \pm 1.32$ \\
\hline
\end{tabular}

The data above show the mean \pm SD. Each of sample size; Elental: $n=263$, Peptino: $n=270$, Ensure H: $n=164$, Maybalance mini: $n=202$, Racol-NF: $n=112$, Maybalance: $n=60$, Tatal: $n=1071$, SD: Standard deviation 
Path analysis model through multiple group SEM

Multiple group SEM was implemented to assess factors that influence the flavor of enteral nutrition agents, according to the model in Fig. 3. The results of individual enteral nutrition analyses were almost the same as the mean value for all enteral nutrition. The impression of taking exhibited a strong and significantly positive path with respect to flavor ( $p<0.001$ ), whereas the feeling of presence exhibited either a weak positive path or significant negative path with respect to flavor $(\mathrm{p}<0.001)$. The lowest $\mathrm{R}^{2}$ value was 0.66 for Elental $^{\circledR}$, which was favorable (Fig. 3).

\section{DISCUSSION}

Conventional reports $[8,9]$ had been evaluated for "bitterness," "sweetness," and "sourness" of enteral nutrients, but in this study, we focused on the flavor of enteral nutrients themselves. From this fact, this

Table 2: Results of a factor analysis of the sensory evaluation of enteral nutrition correlation between the three identified factors and good flavor

\begin{tabular}{|c|c|c|c|}
\hline $\begin{array}{l}\text { Sensory evaluation } \\
\text { items }\end{array}$ & Factor I & Factor II & Factor III \\
\hline Quality of flavor & 0.834 & 0.014 & -0.118 \\
\hline Familiarity & 0.795 & -0.002 & -0.100 \\
\hline $\begin{array}{l}\text { Ease of } \\
\text { consumption }\end{array}$ & 0.790 & 0.023 & -0.036 \\
\hline Consistency & 0.730 & 0.007 & 0.069 \\
\hline Medicinal taste & 0.517 & -0.083 & 0.095 \\
\hline Greasiness & 0.012 & 0.938 & -0.082 \\
\hline Milky smell & -0.033 & 0.697 & 0.011 \\
\hline Positive mouthfeel & -0.012 & 0.467 & 0.211 \\
\hline Persistence of flavor & 0.164 & 0.073 & 0.697 \\
\hline Taste & -0.290 & 0.031 & 0.640 \\
\hline Aftertaste & 0.029 & -0.052 & 0.621 \\
\hline Peculiar flavor & 0.366 & 0.005 & 0.474 \\
\hline $\begin{array}{l}\text { Factor correlation } \\
\text { matrix }\end{array}$ & Factor I & Factor II & Factor III \\
\hline Factor I & - & 0.024 & 0.354 \\
\hline Factor II & & - & 0.470 \\
\hline Factor III & & & - \\
\hline
\end{tabular}

Extraction method: Maximum likelihood, Rotation method: Promax with Kaiser normalization factor loading: Over 0.45 . report revealed the difference in flavor of enteral nutrients, which had not been clarified in the past. A sensory comparison of semi-digestion nutrition agents, in which the primary nitrogen sources are proteins, dipeptides, and tripeptides, and digestion/compositional nutrition agents, which are composed only of crystalline amino acid, revealed that the semi-digestion nutrition agents were rated as having a better flavor, a finding that agreed with previous conventional results [1]. In the flavor comparison, the semi-digestion nutrition agent Meibalance mini ${ }^{\circledR}$ received the significantly highest average score of $3.69(\mathrm{p}<0.001)$, which was near the evaluation point of 4 or "almost good." In contrast, the semi-digestion nutrition agent Meibalance ${ }^{\circledR} 1.0$ received the lowest score, likely because this formula is available in only one flavor and subjects could not choose their preferred flavor. Meibalance mini ${ }^{\circledR}$ was considered a good oral enteral nutrition agent for ensuring adherence to prescribed enteral nutrition. In addition, the finding that Meibalance ${ }^{\circledR}$ 1.0 received the lowest score, despite its classification as a food item, revealed that not all food items have a good flavor. Therefore, because enteral nutrients differ significantly in taste depending on the type, it is important for health-care professionals to fully understand the taste of multiple nutrients and to provide enteral nutrients suitable for the patient's taste.

Regarding the digestion nutrition agents, Elental ${ }^{\circledR}$ received a significantly higher score than Pepuchino ${ }^{\circledR}$ did $(\mathrm{p}<0.001)$. Pepuchino ${ }^{\circledR}$ was rated the lowest of all nutrition agents with a score that approached evaluation point 2, or "almost poor." This suggests that Elental ${ }^{\circledR}$ should be selected over Pepuchino ${ }^{\circledR}$ for the oral administration of enteral nutrition. Again, Elental ${ }^{\circledR}$ likely received a higher score because of the subjects' ability to select their preferred flavor. The factor analyses of 19 evaluation pairs (excluding good flavor) eventually extracted the following subscales: Impression of taking, feeling of presence, and sense of richness.

To assess the influence of these three subscales on flavor, a covariance structure path analysis was implemented in which all nutrition agents were excluded regardless of type. In the final acquired model, the impression of taking exhibited a significantly strong positive path with regard to flavor, whereas the feeling of presence exhibited a weakly path with respect to flavor. In addition, the impression of taking was found to have a dramatic influence on flavor in individual analyses of the enteral nutrition agents. These findings indicate the importance of considering the impression of taking when seeking to improve the flavor of an enteral nutrition agent.

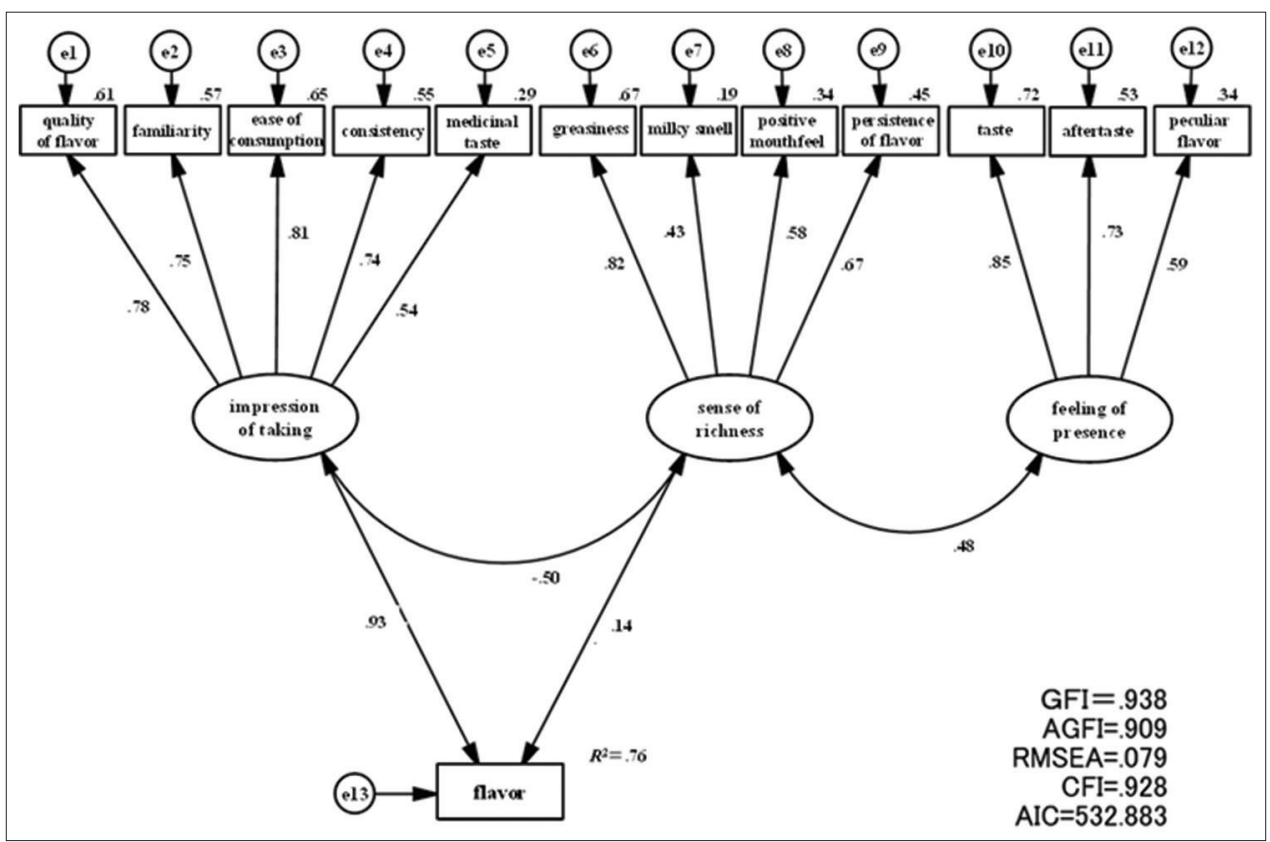

Fig. 2: Path analysis model using the mean values of total enteral nutrition agents 


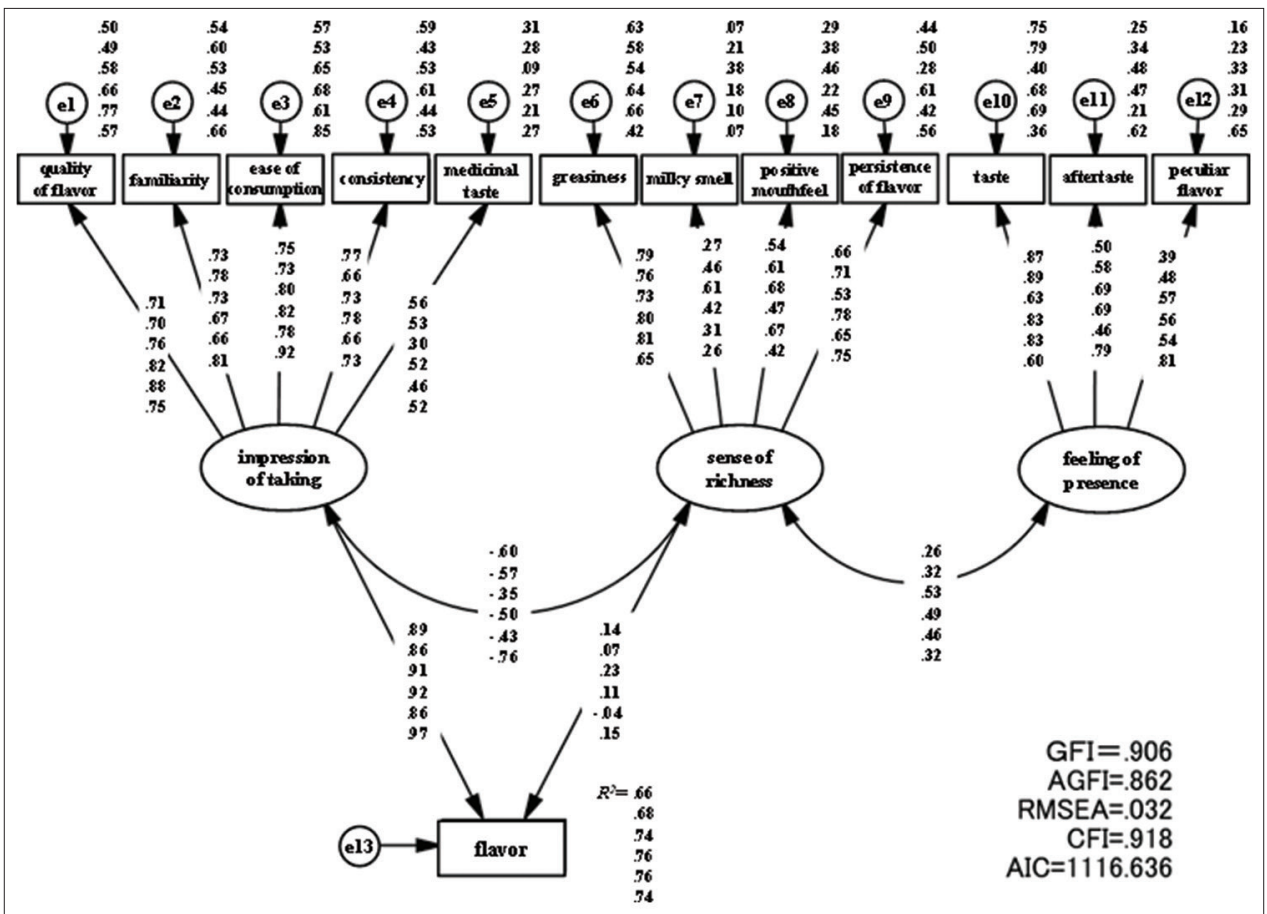

Fig. 3: Multiple group structural equation modeling according to enteral nutrition type. Each value is listed from top to bottom in the order of Elental ${ }^{\circledR}$, Pepuchino $^{\circledR}$, Ensure $^{\circledR} \mathrm{H}$, Meibalance mini $^{\circledR}$, Racol $^{\circledR}$ NF and Meibalance ${ }^{\circledR}$

The participants in this study were college students, and thus the results might not accurately reflect the flavor preferences of patients who are actually receiving enteral nutrition. However, the results of the covariance structure analysis show that the impression of taking influenced the flavor scores of all tested enteral nutrition agents. Therefore, the impression of taking directly influences the flavor of an enteral nutrition agent.

As many studies have been done about the taste of internal medicines $[10,11]$, the flavor of enteral nutrition agent also needs to be studied further in the future.

\section{CONCLUSION}

The results of this study demonstrate the strong influence of impression of taking on the perception of flavor in an enteral nutrition agent. This demonstrates the importance of understanding by medical professionals of the factors that influence the flavors of nutrition agents and the provision of better nutrition agents and instructions to patients, as these factors affect medication adherence.

\section{REFERENCES}

1. Sasaki M. Types and features of enteral formula. JJSPEN 2012;27:637-42.

2. Yamamoto K, Kanetani S, Higashiguchi T, editors. NST Perfect Guide.
Tokyo: Shorinsha; 2009. p. 161-5.

3. Elia M, Ceriello A, Laube H, Sinclair AJ, Engfer M, Stratton RJ. Enteral nutritional support and use of diabetes-specific formulas for patients with diabetes: A systematic review and meta-analysis. Diabetes Care 2005;28(9):2267-79.

4. Heyland DK, Novak F, Drover JW, Jain M, Su X, Suchner U. Should immunonutrition become routine in critically ill patients? A systematic review of the evidence. JAMA 2001;286(8):944-53.

5. Vijayakumar A, Ganesh B. A cross sectional study on patients with enteral nutrition. Int J Pharm Pharm Sci 2013;5(4):101-3.

6. Hanane G, Wafaa D, Hadria G, Benmhel B, Djamel S, Omar K. Symbiotic enhances gut mucosa recovery rate and reduces overgrowth of bacteria in experimental protein malnutrition. Int J Pharm Pharm Sci 2015;7(5):96-100.

7. Osgood EC, Suci GJ, Tannenbaum PH. The Measurement of Meaning. Urbana: University of Illinois Press; 1957.

8. Mukai J, Ishizaka T, Asaka K, Tokuyama E, Tsuji E, Uchida T. Quantitative taste evaluation of semi elemental diets. Jpn J Pharm Health Care Sci 2006;32:383-91.

9. Mukai J, Miyanaga Y, Ishizaka T, Asaka K, Nakai Y, Tsuji E, et al. Quantitative taste evaluation of total enteral nutrients. Chem Pharm Bull (Tokyo) 2004;52(12):1416-21.

10. Sharma V, Chopra H. Role of taste and taste masking of bitter drugs in pharmaceutical industries an overview. Int J Pharm Pharm Sci 2010;2:14-8.

11. Sharma D, Chopra H, Bedi N. Development and evaluation of paracetamol taste masked orally disintegrating tablets using polymer coating technique. Int J Pharm Pharm Sci 2012;4:129-34. 